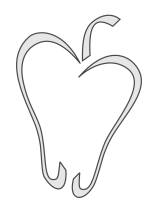

ISSN 1852-4249

\title{
Correlation between asymmetric profiles in slits and standard prewetting lines
}

\author{
Salvador A. Sartarelli, ${ }^{1 *}$ Leszek Szybisz ${ }^{2-4 \dagger}$
}

\begin{abstract}
The adsorption of Ar on substrates of $\mathrm{Li}$ is investigated within the framework of a density functional theory which includes an effective pair potential recently proposed. This approach yields good results for the surface tension of the liquid-vapor interface over the entire range of temperatures, $T$, from the triple point, $T_{t}$, to the critical point, $T_{c}$. The behavior of the adsorbate in the cases of a single planar wall and a slit geometry is analyzed as a function of temperature. Asymmetric density profiles are found for fluid confined in a slit built up of two identical planar walls leading to the spontaneous symmetry breaking (SSB) effect. We found that the asymmetric solutions occur even above the wetting temperature $T_{w}$ in a range of average densities $\rho_{s s b 1}^{*} \leq \rho_{a v}^{*} \leq \rho_{s s b 2}^{*}$, which diminishes with increasing temperatures until its disappearance at the critical prewetting point $T_{c p w}$. In this way a correlation between the disappearance of the SSB effect and the end of prewetting lines observed in the adsorption on a one-wall planar substrate is established. In addition, it is shown that a value for $T_{c p w}$ can be precisely determined by analyzing the asymmetry coefficients.
\end{abstract}

\section{Introduction}

The study of physisorption of fluids on solid substrates had led to very fascinating phenomena mainly determined by the relative strengths of

\footnotetext{
*E-mail: asarta@ungs.edu.ar

${ }^{\dagger}$ E-mail: szybisz@tandar.cnea.gov.ar

1 Instituto de Desarrollo Humano, Universidad Nacional de General Sarmiento, Gutierrez 1150, RA-1663 San Miguel, Argentina.

2 Laboratorio TANDAR, Departamento de Física, Comisión Nacional de Energía Atómica, Av. del Libertador 8250, RA-1429 Buenos Aires, Argentina.

3 Departamento de Física, Facultad de Ciencias Exactas y Naturales, Universidad de Buenos Aires, Ciudad Universitaria, RA-1428 Buenos Aires, Argentina.

4 Consejo Nacional de Investigaciones Científicas y Técnicas, Av. Rivadavia 1917, RA-1033 Buenos Aires, Argentina.
}

fluid-fluid $(f-f)$ and substrate-fluid $(s-f)$ attractions. In the present work we shall refer to two of such features. One is the prewetting curve identified in the study of fluids adsorbed on planar surfaces above the wetting temperature $T_{w}$ (see, e.g., Pandit, Schick, and Wortis [1]) and the other is the occurrence of asymmetric profiles of fluids confined in a slit of identical walls found by van Leeuwen and collaborators in molecular dynamics calculations $[2,3]$. It is known that for a strong substrate (i.e., when the $s-f$ attraction dominates over the $f$ - $f$ one) the adsorbed film builds up continuously showing a complete wetting.In such a case, neither prewetting transitions nor spontaneous symmetry breaking (SSB) of the profiles are observed, both these phenomena appear for substrates of moderate strength.

The prewetting has been widely analyzed for adsorption of quantum as well as classical fluids. A 
Papers in Physics, vol. 1, ARt. 010001 (2009) / S. A. Sartarelli et al.

summary of experimental data and theoretical calculations for ${ }^{4} \mathrm{He}$ may be found in Ref. [4]. Studies of other fluids are mentioned in Ref. [5]. These investigations indicated that prewetting is present in real systems such as ${ }^{4} \mathrm{He}, \mathrm{H}_{2}$, and inert gases adsorbed on alkali metals.

On the other hand, after a recent work of Berim and Ruckenstein [6] there is a renewal of the interest in searching for the SSB effect in real systems. These authors utilized a density functional (DF) theory to study the confinement of $\mathrm{Ar}$ in a slit composed of two identical walls of $\mathrm{CO}_{2}$ and concluded that SSB occurs in a certain domain of temperatures. In a revised analysis of this case, reported in Ref. [7], we found that the conditions for the SSB were fulfilled because the authors of Ref. [6] had diminished the $s$ - $f$ attraction by locating an extra hard-wall repulsion. However, it was found that inert gases adsorbed on alkali metals exhibit SSB. Results for Ne confined by such substrates were recently reported [8].

The aim of the present investigation is to study the relation between the range of temperatures where the SSB occurs and the temperature dependence of the wetting properties. In this paper we illustrate our findings describing the results for $\mathrm{Ar}$ adsorbed on Li. Previous DF calculations of Ancilotto and Toigo [9] as well as Grand Canonical Monte Carlo (GCMC) simulations carried out by Curtarolo et al. [10] suggest that $\mathrm{Ar}$ wets $\mathrm{Li}$ at a temperature significantly below $T_{c}$. So, this system should exhibit a large locus of the prewetting line and this feature makes it very convenient for our study as it was already communicated during a recent workshop [11].

The paper is organized in the following way. The theoretical background is summarized in Sec. II. The results, together with their analysis, are given in Sec. III.. Sec. IV. is devoted to the conclusions.

\section{Theoretical background}

In a DF theory, the Helmholtz free energy $F_{\mathrm{DF}}[\rho(\mathbf{r})]$ of an inhomogeneous fluid embedded in an external potential $U_{s f}(\mathbf{r})$ is expressed as a functional of the local density $\rho(\mathbf{r})$ (see, e.g., Ref. [12])

$$
\begin{aligned}
& F_{\mathrm{DF}}[\rho(\mathbf{r})] \\
= & \nu_{\mathrm{id}} k_{B} T \int d \mathbf{r} \rho(\mathbf{r})\left\{\ln \left[\Lambda^{3} \rho(\mathbf{r})\right]-1\right\} \\
+ & \int d \mathbf{r} \rho(\mathbf{r}) f_{\mathrm{HS}}\left[\bar{\rho}(\mathbf{r}) ; d_{\mathrm{HS}}\right] \\
+ & \frac{1}{2} \iint d \mathbf{r} d \mathbf{r} / \rho(\mathbf{r}) \rho(\mathbf{r} / \prime) \Phi_{\mathrm{attr}}(|\mathbf{r}-\mathbf{r} /|) \\
+ & \int d \mathbf{r} \rho(\mathbf{r}) U_{s f}(\mathbf{r}) .
\end{aligned}
$$

The first term is the ideal gas free energy, where $k_{B}$ is the Boltzmann constant and $\Lambda=$ $\sqrt{2 \pi \hbar^{2} / m k_{B} T}$ the de Broglie thermal wavelength of the molecule of mass $m$. Quantity $\nu_{\text {id }}$ is a parameter introduced in Eq. (2) of [13] (in the standard theory it is equal unity). The second term accounts for the repulsive $f$ - $f$ interaction approximated by a hard-sphere (HS) functional with a certain choice for the HS diameter $d_{\mathrm{HS}}$. In the present work we have used for $f_{\mathrm{HS}}\left[\bar{\rho}(\mathbf{r}) ; d_{\mathrm{HS}}\right]$ the expression provided by the nonlocal DF (NLDF) formalism developed by Kierlik and Rosinberg [14] (KR), where $\bar{\rho}(\mathbf{r})$ is a properly averaged density. The third term is the attractive $f$ - $f$ interactions treated in a mean field approximation (MFA). Finally, the last integral represents the effect of the external potential $U_{s f}(\mathbf{r})$ exerted on the fluid.

In the present work, for the analysis of physisorption we adopted the $a b$ initio potential of Chismeshya, Cole, and Zaremba (CCZ) [15] with the parameters listed in Table 1 therein.

\section{i. Effective pair attraction}

The attractive part of the $f$ - $f$ interaction was described by an effective pair interaction devised in Ref. [5], where the separation of the Lennard-Jones (LJ) potential introduced by Weeks, Chandler and Andersen (WCA) [16] is adopted

$$
\begin{aligned}
& \Phi_{\mathrm{attr}}^{\mathrm{WCA}}(r) \\
= & \begin{cases}-\tilde{\varepsilon}_{f f} \\
4 \tilde{\varepsilon}_{f f}\left[\left(\frac{\tilde{\sigma}_{f f}}{r}\right)^{12}-\left(\frac{\tilde{\sigma}_{f f}}{r}\right)^{6}\right], & r>r_{m} .\end{cases}
\end{aligned}
$$


Here $r_{m}=2^{1 / 6} \tilde{\sigma}_{f f}$ is the position of the LJ minimum. No cutoff for the pair potential was introduced. The well depth $\tilde{\varepsilon}_{f f}$ and the interaction size $\tilde{\sigma}_{f f}$ are considered as free parameters because the use of the bare values $\varepsilon_{f f} / k_{B}=119.76 \mathrm{~K}$ and $\sigma_{f f}=3.405 \AA$ overestimates $T_{c}$.

So, the complete DF formalism has three adjustable parameters (namely, $\nu_{i d}, \tilde{\varepsilon}_{f f}$, and $\tilde{\sigma}_{f f}$ ), which were determined by imposing that at $l-v$ coexistence, the pressure as well as the chemical potential of the bulk $l$ and $v$ phases should be equal [i.e., $P\left(\rho_{l}\right)=P\left(\rho_{v}\right)$ and $\mu\left(\rho_{l}\right)=\mu\left(\rho_{v}\right)$ ]. The procedure is described in Ref. [5]. In practice, we set $d_{\mathrm{HS}}=\tilde{\sigma}_{f f}$ and imposed the coexistence data of $\rho_{l}$, $\rho_{v}$, and $P\left(\rho_{l}\right)=P\left(\rho_{v}\right)=P_{0}$ for Ar quoted in Table X of Ref. [17] to be reproduced in the entire range of temperatures $T$ between $T_{t}=83.78 \mathrm{~K}$ and $T_{c}=150.86 \mathrm{~K}$.

\section{ii. Euler-Lagrange equation}

The equilibrium density profile $\rho(\mathbf{r})$ of the adsorbed fluid is determined by a minimization of the free energy with respect to density variations with the constraint of a fixed number of particles $N$

$$
\frac{\delta}{\delta \rho(\mathbf{r})}\left[F_{\mathrm{DF}}[\rho(\mathbf{r})]-\mu \int d \mathbf{r} \rho(\mathbf{r})\right]=0 .
$$

Here the Lagrange multiplier $\mu$ is the chemical potential of the system. In the case of a planar symmetry where the flat walls exhibit an infinite extent in the $x$ and $y$ directions, the profile depends only on the coordinate $z$ perpendicular to the substrate. For this geometry, the variation of Eq. (3) yields the following Euler-Lagrange (E-L) equation

$$
\begin{aligned}
\frac{\delta\left[\left(F_{\mathrm{id}}+F_{\mathrm{HS}}\right) / A\right]}{\delta \rho(z)} & +\int_{0}^{L} d z \prime \rho(z \prime) \bar{\Phi}_{\mathrm{attr}}(|z-z \prime|) \\
& +U_{s f}(z)=\mu,
\end{aligned}
$$

where

$$
\frac{\delta\left(F_{\mathrm{id}} / A\right)}{\delta \rho(z)}=\nu_{\mathrm{id}} k_{B} T \ln \left[\Lambda^{3} \rho(z)\right],
$$

and

$$
\begin{gathered}
\frac{\delta\left(F_{\mathrm{HS}} / A\right)}{\delta \rho(z)}=f_{\mathrm{HS}}\left[\bar{\rho}(z) ; d_{\mathrm{HS}}\right] \\
+\quad \int_{0}^{L} d z \prime \rho(z \prime) \frac{\delta f_{\mathrm{HS}}\left[\bar{\rho}(z \prime) ; d_{\mathrm{HS}}\right]}{\delta \bar{\rho}(z \prime)} \frac{\delta \bar{\rho}(z \prime)}{\delta \rho(z)} .
\end{gathered}
$$

Here $F_{\text {id }} / A$ and $F_{\mathrm{HS}} / A$ are free energies per unit of one wall area $A$. $L$ is the size of the box adopted for solving the E-L equations. The boundary conditions for the one-wall and slit systems are different and will be given below. The final E-L equation may cast into the form

$$
\nu_{\text {id }} k_{B} T \ln \left[\Lambda^{3} \rho(z)\right]+Q(z)=\mu,
$$

where

$$
\begin{aligned}
Q(z) & =f_{\mathrm{HS}}\left[\bar{\rho}(z) ; d_{\mathrm{HS}}\right] \\
& +\int_{0}^{L} d z \prime \rho(z \prime) \frac{\delta f_{\mathrm{HS}}\left[\bar{\rho}(z \prime) ; d_{\mathrm{HS}}\right]}{\delta \bar{\rho}(z \prime)} \frac{\delta \bar{\rho}(z \prime)}{\delta \rho(z)} \\
& +\int_{0}^{L} d z \prime \rho(z \prime) \bar{\Phi}_{\mathrm{attr}}(|z-z \prime|) \\
& +U_{s f}(z) .
\end{aligned}
$$

The number of particles $N_{s}$ per unit area, $A$, of the wall is

$$
N_{s}=\frac{N}{A}=\int_{0}^{L} \rho(z) d z .
$$

In order to get solutions for $\rho(z)$, it is useful to rewrite Eq. (7) as

$$
\rho(z)=\rho_{0} \exp \left(-\frac{Q(z)}{\nu_{\mathrm{id}} k_{B} T}\right),
$$

with

$$
\rho_{0}=\frac{1}{\Lambda^{3}} \exp \left(\frac{\mu}{\nu_{\text {id }} k_{B} T}\right) .
$$

The relation between $\mu$ and $N_{s}$ is obtained by substituting Eq. (10) into the constraint of Eq. (9)

$$
\begin{aligned}
\mu & =-\nu_{\mathrm{id}} k_{B} T \\
& \times \ln \left[\frac{1}{N_{s} \Lambda^{3}} \int_{0}^{L} d z \exp \left(-\frac{Q(z)}{\nu_{\mathrm{id}} k_{B} T}\right)\right]
\end{aligned}
$$

When solving this kind of systems, it is usual to define dimensionless variables $z^{*}=z / \tilde{\sigma}_{f f}$ for the distance and $\rho^{*}=\rho \tilde{\sigma}_{f f}^{3}$ for the densities. In these units the box size becomes $L^{*}=L / \tilde{\sigma}_{f f}$. 
PAPers in Physics, vol. 1, ART. 010001 (2009) / S. A. Sartarelli et al.

\section{Results and Analysis}

In order to quantitatively study the adsorption of fluids within any theoretical approach,one must require the experimental surface tension of the bulk liquid-vapor interface, $\gamma_{l v}$, to be reproduced satisfactorily over the entire $T_{t} \leq T \leq T_{c}$ temperature range. Therefore, we shall first examine the prediction for this observable before studying the adsorption phenomena.

\section{i. Surface tension of the bulk liquid-vapor interface}

Figure 1 shows the experimental data of $\gamma_{l v}$ taken from Table II of Ref. [18]. In order to theoretically evaluate this quantity the E-L equations for free slabs of Ar, i.e. setting

$$
U_{s f}(z)=0,
$$

were solved imposing periodic boundary conditions $\rho(z=0)=\rho(z=L)$. At a given temperature $T$, for a sufficiently large system one must obtain a wide central region with $\rho(z \simeq L / 2)=\rho_{l}(T)$ and tails with density $\rho_{v}(T)$, where the values of $\rho_{l}(T)$ and $\rho_{v}(T)$ should be those of the liquid-vapor coexistence curve. The surface tension of the liquid-vapor interface is calculated according to the thermodynamic definition

$$
\gamma_{l v}=\left(\Omega+P_{0} V\right) / A=\Omega / A+P_{0} L,
$$

where $\Omega=F_{\mathrm{DF}}-\mu N$ is the grand potential of the system and $P_{0}$ the pressure at liquid-vapor coexistence previously introduced. We solved a box with $L^{*}=40$. The obtained results are plotted in Fig. 1 together with the prediction of the fluctuation theory of critical phenomena $\gamma_{l v}=\gamma_{l v}^{0}\left(1-T / T_{c}\right)^{1.26}$ with $\gamma_{l v}^{0}=17.4 \mathrm{~K} / \AA^{2}$ (see, e.g., [19]). One may realize that our values are in satisfactory agreement with experimental data and the renormalization theory over the entire range of temperatures $T_{t} \leq T \leq T_{c}$, showing a small deviation near $T_{t}$.

\section{ii. Adsorption on one planar wall}

It is assumed that the physisorption of Ar on a one wall substrate of $\mathrm{Li}$ is driven by the CCZ potential, i.e.,

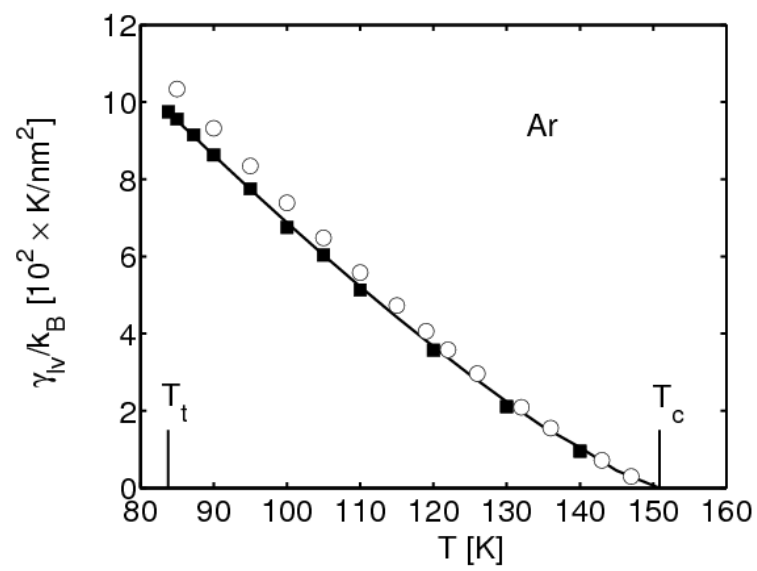

Figure 1: Surface tension of Ar as a function of temperature. Squares are experimental data taken from Table II of Ref. [18]. The solid curve corresponds to the fluctuation theory of critical phenomena and the circles are present DF results.

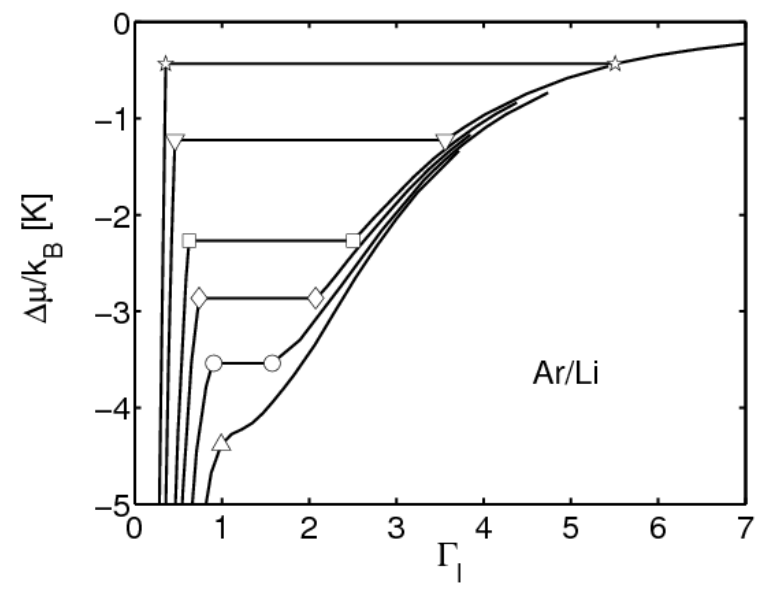

Figure 2: Adsorption isotherms for the Ar/Li system, i.e., $\Delta \mu$ as a function of coverage $\Gamma_{\ell}$. Uptriangles correspond to $T=119 \mathrm{~K}$; circles to $T=118 \mathrm{~K}$; diamonds to $T=117 \mathrm{~K}$; squares to $T=116 \mathrm{~K}$; down-triangles to $T=114 \mathrm{~K}$ and stars to $T=112 \mathrm{~K}$.

$$
U_{s f}(z)=U_{\mathrm{CCZ}}(z) .
$$

The E-L equations were solved in a box of size $L^{*}=40$ by imposing $\rho(z>L)=\rho(z=L)$. The solution gives a density profile $\rho(z)$ and the corresponding chemical potential $\mu$. Adsorption 
isotherms at a given temperature were calculated as function of the excess surface density. This quantity, also termed coverage, is often expressed in nominal layers $\ell$

$$
\Gamma_{\ell}=\left(1 / \rho_{l}^{2 / 3}\right) \int_{0}^{\infty} d z\left[\rho(z)-\rho_{B}\right]
$$

where $\rho_{B}=\rho(z \rightarrow \infty)$ is the asymptotic bulk density and $\rho_{l}$ the liquid density at saturation for a given temperature. By utilizing the results for $\mu$ obtained from the E-L equation and the value $\mu_{0}$ corresponding to saturation at a given temperature $T$, the difference $\Delta \mu=\mu-\mu_{0}$ was evaluated. Figure 2 shows the adsorption isotherms for temperatures above $T_{w}$, where an equal area Maxwell construction is feasible. This is just the prewetting region characterized by a jump in coverage $\Gamma_{\ell}$. The size of this jump depends on temperate. The largest jump occurs at $T_{w}$ and diminishes for increasing $T$ until its disappearance at $T_{c p w}$. Density profiles just below and above the coverage jump for $T=114 \mathrm{~K}$ are displayed in Fig. 3 , in that case $\Gamma_{\ell}$ jumps from 0.5 to 3.6. Therefore, the formation of the fourth layer may be observed in the plot.

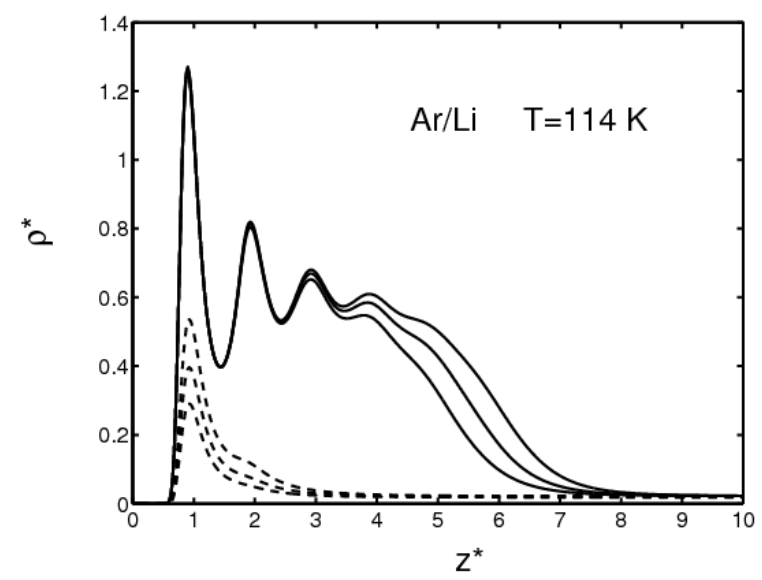

Figure 3: Examples of density profiles of Ar adsorbed on a surface of $\mathrm{Li}$ at $T=114 \mathrm{~K}$ displayed as a function of the distance from the wall located at $z^{*}=0$. Dashed curves are profiles for $\Gamma_{\ell}$ below the coverage jump, while solid curves are stable films above this jump.

The wetting temperature $T_{w}$ can be obtained from the analysis of the values of $\Delta \mu / k_{B}$ at which

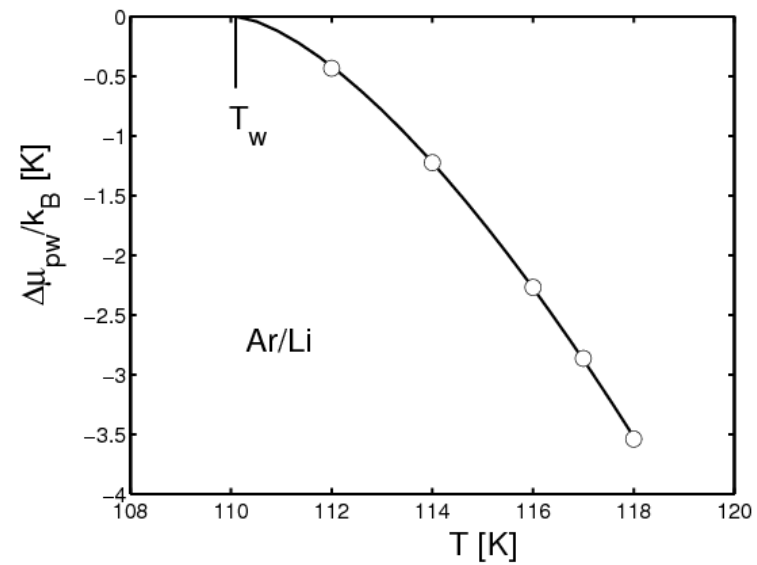

Figure 4: Prewetting line for Ar adsorbed on Li. The solid curve is the fit to Eq. (17) and reaches the $\Delta \mu_{p w} / k_{B}=0$ line at $T_{w}=110.1 \mathrm{~K}$.

the jump in coverage occurs at each considered temperature. The behavior $\Delta \mu_{p w} / k_{B}$ vs T is displayed in Fig. 4. A useful form for determining the temperature $T_{w}$ was derived from thermodynamic arguments $[20]$

$$
\begin{aligned}
\Delta \mu_{p w}(T) & =\mu_{p w}(T)-\mu_{0}(T) \\
& =a_{p w}\left(T-T_{w}\right)^{3 / 2}
\end{aligned}
$$

Here $a_{p w}$ is a model parameter and the exponent $3 / 2$ is fixed by the power of the van der Walls tail of the adsorption potential $U_{s f}(z) \simeq-C_{3} / z^{3}$. The fit of the data of $\Delta \mu / k_{B}$ to Eq. (17) yielded $T_{w}=$ $110.1 \mathrm{~K}$ and $a_{p w} / k_{B}=-0.16 \mathrm{~K}^{-1 / 2}$.

On the other hand, according to Fig. 2, the critical prewetting point $T_{c p w}$ lies between $T=118$ and $119 \mathrm{~K}$. At the latter temperature, the film already presents a continuous growth.

Our values of $T_{w}$ and $T_{c p w}$ are smaller than those obtained from prior DF calculations $[9]\left(T_{w}=\right.$ $123 \mathrm{~K}$ and $T_{c p w} \simeq 130 \mathrm{~K}$ ) and GCMC simulations [10] $\left(T_{w}=130 \mathrm{~K}\right)$. The difference with the DF evaluation of Ref. [9] is due to the use of different effective pair potentials as we explain in Ref. [5], where the adsorption of Ne is studied. The present approach gives a reasonable $\gamma_{l v}$, while that of Ref. [9] fails dramatically close to $T_{t}$. The difference with the GCMC results cannot be interpreted in a straightforward way. 
Papers in Physics, vol. 1, ARt. 010001 (2009) / S. A. Sartarelli et al.

\section{iii. Confinement in a planar slit}

In the slit geometry, where the Ar atoms are confined by two identical walls of Li the $s-f$ potential becomes

$$
U_{s f}(z)=U_{\mathrm{CCZ}}(z)+U_{\mathrm{CCZ}}(L-z) .
$$

The walls were located at a distance $L^{*}=40$, this width guarantees that the pair interaction between two atoms located at different walls is negligible. In fact, this width is wider than $L^{*}=29.1$, which was utilized in the pioneering molecular dynamics calculations $[2,3]$. Accordingly, the E-L equations were solved in a box of size $L^{*}=40$. In this geometry, the repulsion at the walls causes the profiles $\rho(z=0)$ and $\rho(z=L)$ to be equal to zero. The solutions were obtained at a fixed dimensionless average density defined in terms of $N, A$, and $L$ as $\rho_{a v}^{*}=N \tilde{\sigma}_{f f}^{3} / A L=N_{s}^{*} / L^{*}$.

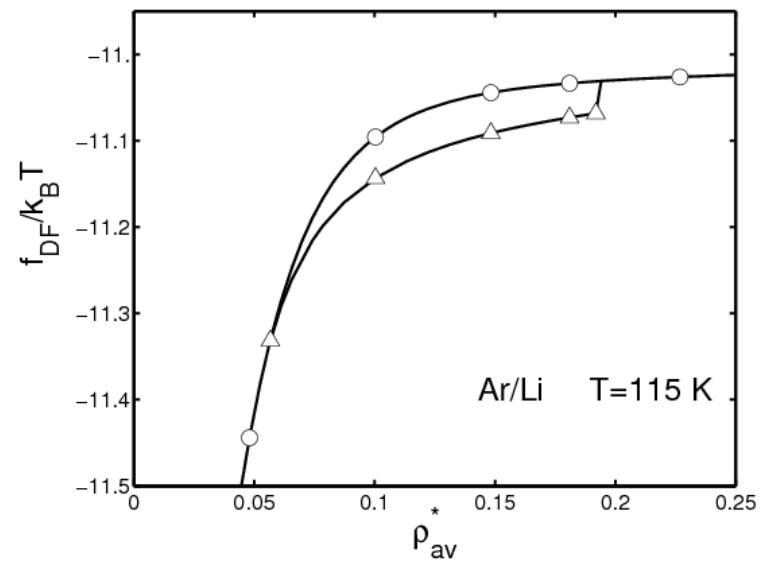

Figure 5: Free energy per particle (in units of $k_{B} T$ ) for Ar confined in a slit of $\mathrm{Li}$ with $L^{*}=40$ at $T=115 \mathrm{~K}$ displayed as a function of the average density. The curve labeled by circles corresponds to symmetric solutions, while that labeled by triangles corresponds to asymmetric ones. The SSB occurs in a certain range of average density $\rho_{s s b 1}^{*} \leq \rho_{a v}^{*} \leq$ $\rho_{s s b 2}^{*}$.

For temperatures below $T_{w}=110.1 \mathrm{~K}$, we obtained large ranges of $\rho_{a v}^{*}$ where the asymmetric solutions exhibit a lower free energy than the corresponding symmetric ones. In spite of the fact that there is a general idea that a connection exists between the SSB effect and nonwetting, we have found, by contrast, that SSB behavior extends above the wetting temperature. Furthermore, we have also found a relation between prewetting and SSB.

Figure 5 shows the free energy per particle, $f_{\mathrm{DF}}=F_{\mathrm{DF}} / N$, for both symmetric and asymmetric solutions for the $\mathrm{Ar} / \mathrm{Li}$ system at $T=115 \mathrm{~K}>T_{w}$ as a function of the average density. According to this picture, the ground state (g-s) exhibits asymmetric profiles between a lower and an upper limit $\rho_{s s b 1}^{*}=0.057 \leq \rho_{a v}^{*} \leq \rho_{s s b 2}^{*}=0.192$. Out of this range no asymmetric solutions were obtained form the set of Eqs. (7)-(12). Similar features were obtained for higher temperatures until $T=118 \mathrm{~K}$, above this value the profiles corresponding to the g-s are always symmetric. Figure 6 shows three examples of solutions determined at $T=115 \mathrm{~K}$. The result labeled 1 is a small asymmetric profile, that labeled 2 is the largest asymmetric solution at this temperature. So, by further increasing $\rho_{a v}^{*}$, the SSB effect disappears and the $g$-s becomes symmetric, as indicated by the curve labeled 3 . When the asymmetric profiles occur, the situation is denoted as partial (or one wall) wetting. The symmetric solutions account for a complete (two wall) wetting. These different situations can be interpreted in terms of the balance of $\gamma_{s l}, \gamma_{s v}$ and $\gamma_{l v}$ surface tensions, carefully discussed in previous works $[2,3,7]$. Here we shall restrict ourselves to briefly outline the main features. When the liquid is adsorbed symmetrically like in the case of profile 3 in Fig. 6, there are two $s-l$ and two $l-v$ interfaces. Hence, the total surface excess energy may be written as

$$
\gamma_{t o t}^{s y m}=2 \gamma_{s l}+2 \gamma_{l v} .
$$

On the other hand, for a asymmetric profile $\gamma_{t o t}^{a s y}$ becomes

$$
\gamma_{t o t}^{a s y}=\gamma_{s l}+\gamma_{l v}+\gamma_{s v} .
$$

The three quantities of the r.h.s. of this equation are related by Young's law (see, e.g., Eq. (2.1) in Ref. [21])

$$
\gamma_{s v}=\gamma_{s l}+\gamma_{l v} \cos \theta,
$$

where $\theta$ is the contact angle defined as the angle between the wall and the interface between the liquid and the vapor (see Fig. 1 in Ref. [21]). By using Young's law, the Eq. (20) may be rewritten as 


$$
\gamma_{t o t}^{a s y}=2 \gamma_{s l}+\gamma_{l v}(1+\cos \theta),
$$

with $\cos \theta=\left(\gamma_{s v}-\gamma_{s l}\right) / \gamma_{l v}<1$. If one changes $\gamma_{s l}$ by increasing enough $N_{s}$ (as shown in Fig. 5), and/or $T$, and/or the strength of $U_{s f}(z)$, eventually the equality $\gamma_{s v}-\gamma_{s l}=\gamma_{l v}$ may be reached yielding $\cos \theta=1$. Then, the system would undergo a transition to a symmetric profile where both walls of the slit are wet.

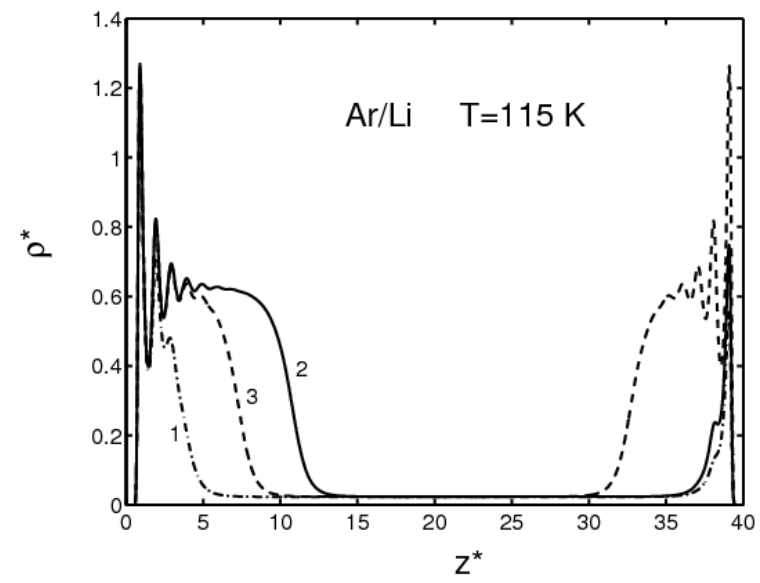

Figure 6: Density profiles of Ar confined in a slit of $\mathrm{Li}$ with $L^{*}=40$ at $T=115 \mathrm{~K}$. The displayed spectra denoted by 1,2 and 3 correspond to average densities $\rho_{a v}^{*}=0.074,0.192$ and 0.218 , respectively.

It is important to remark that, indeed, there are two degenerate asymmetric solutions. Besides that one shown in Fig. 6 where the profiles exhibit the thicker film adsorbed on the left wall (left asymmetric solutions - LAS), there is an asymmetric solution with exactly the same free energy but where the thicker film is located near the right wall (right asymmetric solutions - RAS).

The asymmetry of density profiles may be measured by the quantity

$$
\Delta_{N}=\frac{1}{N_{s}} \int_{0}^{L / 2} d z[\rho(z)-\rho(L-z)] .
$$

According to this definition, if the profile is completely asymmetrical about the middle of the slit, i.e. for: (i) $\rho(z<L / 2) \neq 0$ and $\rho(z \geq L / 2)=0$; or (ii) $\rho(z<L / 2)=0$ and $\rho(z \geq L / 2) \neq 0$ this

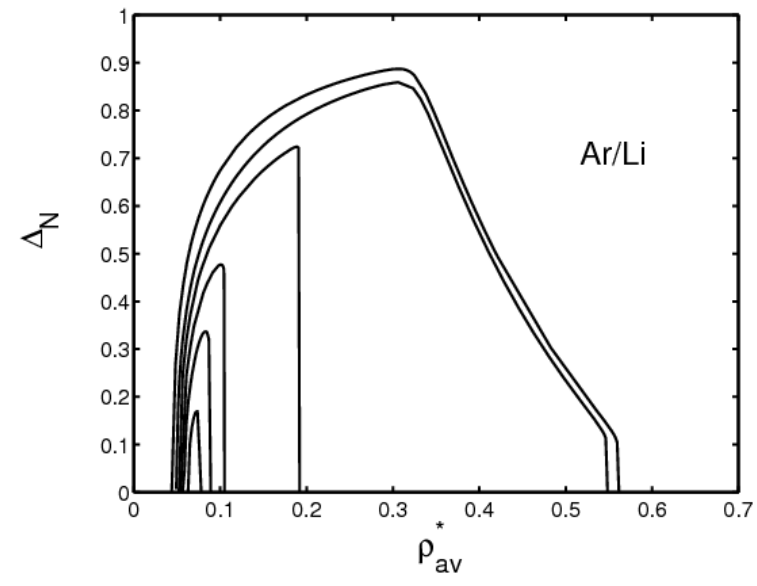

Figure 7: Asymmetry parameter for Ar confined by two Li walls separated by a distance of $L^{*}=40$ as a function of average density. From outside to inside the curves correspond to temperatures $T=$ $112,114,115,116,117$ and $118 \mathrm{~K}$. The asymmetric solutions occur for different ranges $\rho_{s s b 1}^{*} \leq \rho_{a v}^{*} \leq$ $\rho_{s s b 2}^{*}$.

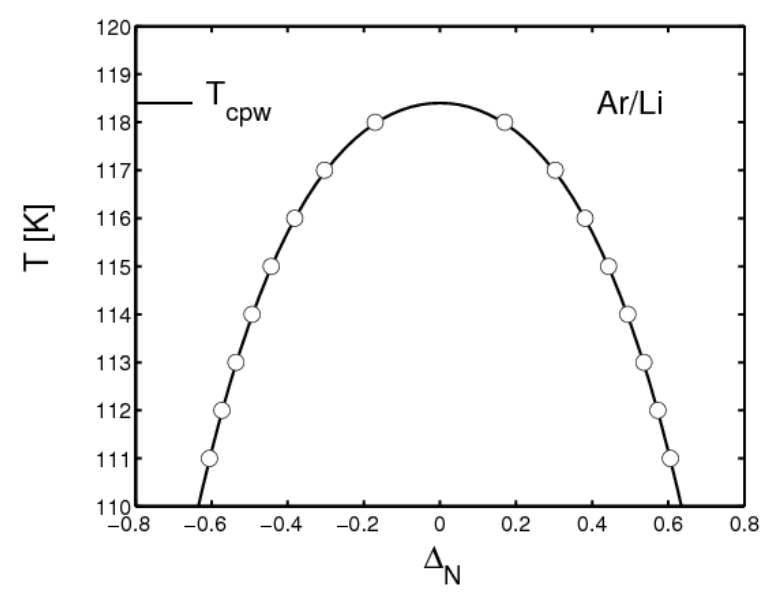

Figure 8: Circles stand for both branches of the asymmetry parameter for Ar confined in an $L^{*}=$ 40 slit of Li walls for temperatures between $T_{w}$ and $T_{c p w}$. The solid curve is the fit to Eq. (24) used to determine $T_{c p w}$.

quantity becomes +1 or -1 , respectively, while for symmetric solutions it vanishes.

We evaluated the asymmetry coefficients of solutions obtained for increasing temperatures up to $T=118 \mathrm{~K}$. The results for LAS profiles at tem- 
Papers in Physics, vol. 1, ARt. 010001 (2009) / S. A. Sartarelli et al.

peratures larger that $T_{w}$ are displayed in Fig. 7 as a function of the average density. One may observe how the range $\rho_{s s b 1}^{*} \leq \rho_{a v}^{*} \leq \rho_{s s b 2}^{*}$ diminishes under increasing temperatures. The SSB effect persists at most for the critical $\rho_{a v}^{*}($ crit $)=$ $(17 / 24) \tilde{\sigma}_{f f}^{2} \times 10^{-2} \simeq 0.074$ with $\tilde{\sigma}_{f f}$ expressed in A.

We shall demonstrate that by analyzing the data of $\Delta_{N}$ for $\rho_{a v}^{*}($ crit $)$ it is possible to determine the critical prewetting point. Figure 8 shows these values for both the LAS and RAS profiles, calculated at different temperatures, suggesting a rather parabolic shape. So, we propose a fit to the following quartic polynomial

$$
T=T_{c p w}+a_{2} \Delta_{N}^{2}+a_{4} \Delta_{N}^{4} .
$$

This procedure yielded $T_{c p w}=118.4 \mathrm{~K}, a_{2}=$ $-14.14 \mathrm{~K}$, and $a_{4}=-16.63 \mathrm{~K}$. The obtained value of $T_{c p w}$ is in agreement with the limits established when analyzing the adsorption isotherms of the one-wall systems displayed in Fig. 2. These results indicate that the disappearance of the SSB effect coincides with the end of the prewetting line.

\section{Conclusions}

We have performed a consistent study within the same DF approach of free slabs of Ar, the adsorption of these atoms on a single planar wall of $\mathrm{Li}$ and its confinement in slits of this alkali metal. Good results were obtained for the surface tension of the liquid-vapor interface. The analysis of the physisorption on a planar surface indicates that Ar wets surfaces of $\mathrm{Li}$ in agreement with previous investigations. The isotherms for the adsorption on one planar wall exhibit a locus of prewetting in the $\mu-T$ plane. A fit of such data yielded a wetting temperature $T_{w}=110.1 \mathrm{~K}$. In addition, these isotherms also show that the critical prewetting point $T_{c p w}$ lies between $T=118$ and $119 \mathrm{~K}$. These results for $T_{w}$ and $T_{c p w}$ are slightly below the values obtained in Refs. $[9,10]$, the discrepancy is discussed in the text.

On the other hand, this investigation shows that the profiles of Ar confined in a slit of Li present SSB. This effect occurs in a certain range of average densities $\rho_{s s b 1}^{*} \leq \rho_{a v}^{*} \leq \rho_{s s b 2}^{*}$, which diminishes for increasing temperatures. The main output of this work is the finding that above the wetting temperature the SSB occurs until $T_{c p w}$ is reached. To the best of our knowledge this is the first time that such a correlation is reported. Furthermore, it is shown that by examining the evolution of the asymmetry coefficient one can precisely determine $T_{c p w}$. The obtained value $T_{c p w}=118.4 \mathrm{~K}$ lies in the interval established when analyzing the adsorption on a single wall.

Acknowledgements - This work was supported in part by the Grants PICT 31980/5 from Agencia Nacional de Promoción Científica y Tecnológica, and X099 from Universidad de Buenos Aires, Argentina.

[1] R Pandit, M Schick, M Wortis, Systematics of multilayer adsorption phenomena on attractive substrates Phys. Rev. B 26, 5112 (1982).

[2] J H Sikkenk, J O Indekeu, J M J van Leeuwen, E O Vossnack, Molecular-dynamics simulation of wetting and drying at solid-fluid interfaces Phys. Rev. Lett. 59, 98 (1987).

[3] M J P Nijmeijer, C Bruin, A F Bakker, J M J van Leeuwen, Wetting and drying of an inert wall by a fluid in a molecular-dynamics simulation, Phys. Rev. A 42, 6052 (1990).

[4] L Szybisz, Adsorption of superfluid ${ }^{4} \mathrm{He}$ films on planar heavy-alkali metals studied with the Orsay-Trento density functional, Phys. Rev. B 67, 132505 (2003).

[5] S A Sartarelli, L Szybisz, I Urrutia, Adsorption of $\mathrm{Ne}$ on alkali surfaces studied with a density functional theory, Phys. Rev. E 79, 011603 (2009).

[6] G O Berim, E Ruckenstein, Symmetry breaking of the fluid density profiles in closed nanoslits, J. Chem. Phys. 126, 124503 (2007).

[7] L Szybisz, S A Sartarelli, Density profiles of Ar adsorbed in slits of $\mathrm{CO}_{2}$ : Spontaneous symmetry breaking revisited, J. Chem. Phys. 128, 124702 (2008). 
PAPers in Physics, vol. 1, ART. 010001 (2009) / S. A. Sartarelli et al.

[8] S A Sartarelli, L Szybisz, I Urrutia, Spontaneous symmetry breaking and first-order phase transitions of adsorbed fluids, Int. J. Bifurcation Chaos (in press).

[9] F Ancilotto, F Toigo, Prewetting transitions of $\mathrm{Ar}$ and $\mathrm{Ne}$ on alkali-metal surfaces surface, Phys. Rev. B 60, 9019 (1999).

[10] S Curtarolo, G Stan, M J Bojan, M W Cole, $\mathrm{W}$ A Steele, Threshold criterion for wetting at the triple point, Phys. Rev. E 61, 1670 (2000).

[11] L Szybisz and S A Sartarelli, Adsorción de gases nobles sobre sustratos planos de metales alcalinos, Communication at the Workshop TREFEMAC09 held at the Univerisidad Nacional de La Pampa, Santa Rosa, Argentina, May 4-6 (2009).

[12] P I Ravikovitch, A Vishnyakov, A V Neimark, Density functional theories and molecular simulations of adsorption and phase transitions in nanopores, Phys. Rev. E 64, 011602 (2001).

[13] F Ancilotto, S Curtarolo, F Toigo, M W Cole, Evidence concerning drying behavior of $\mathrm{Ne}$ near a Ce surface, Phys. Rev. Lett. 87, 206103 (2001).

[14] E Kierlik, M L Rosinberg, Free-energy density functional for the inhomogeneous hardsphere fluid: Application to interfacial adsorption, Phys. Rev. A 42, 3382 (1990).
[15] A Chizmeshya, M W Cole, E. Zaremba, Weak biding potentials and wetting transitions, J. Low Temp. Phys. 110, 677 (1998).

[16] J D Weeks, D Chandler, H C Andersen, Role of repulsive forces in determining the equilibrium structure of simple fluids, J. Chem. Phys. 54, 5237 (1971).

[17] V A Rabinovich, A A Vasserman, V I Nedostup, L S Veksler, Thermophysical properties of neon, argon, krypton and xenon, Hemisphere, Washington DC (1988).

[18] S-T Wu, G-S Yan, Surface tensions of simple liquids, J. Chem. Phys. 77, 5799 (1982).

[19] J Vrabec, G K Kedia, G Fuchs, H Hasse, Vapour-liquid coexistence of the truncated and shifted Lennard-Jones fluid, Mol. Phys. 104, 1509 (2006).

[20] E Cheng, G Mistura, H C Lee, M H W Chan, M W Cole, C Carraro, W F Saam, F Toigo, Wetting transitions of liquid hydrogen films, Phys. Rev. Lett. 70, 1854 (1993).

[21] P G de Gennes, Wetting: statics and dynamics, Rev. Mod. Phys. 57, 827 (1985). 